Pacific

Journal of

Mathematics

AN ELECTROSTATICS MODEL FOR ZEROS OF GENERAL ORTHOGONAL POLYNOMIALS

Mourad E.H. IsMaIL 


\title{
AN ELECTROSTATICS MODEL FOR ZEROS OF GENERAL ORTHOGONAL POLYNOMIALS
}

\author{
MOURAD E.H. ISMAIL
}

We prove that the zeros of general orthogonal polynomials, subject to certain integrability conditions on their weight functions determine the equilibrium position of movable $n$ unit charges in an external field determined by the weight function. We compute the total energy of the system in terms of the recursion coefficients of the orthonormal polynomials and study its limiting behavior as the number of particles tends to infinity in the case of Freud exponential weights.

\section{Introduction.}

Stieltjes [24], [25] considered the following electrostatic model. Fix two charges $(\alpha+1) / 2$ and $(\beta+1) / 2$ at $x=1$ and $x=-1$, respectively, then put $n$ movable unit charges at distinct points in $(-1,1)$. The question is to determine the equilibrium position of the movable charges when the interaction forces arise from a logarithmic potential. Stieltjes proved that the equilibrium position is attained at the zeros of the Jacobi polynomial $P_{n}^{(\alpha, \beta)}(x)$. For a proof see Szegó's book [26]. Another electrostatic problem is to have a fixed point charge $(\alpha+1) / 2$ at $x=0$ and $n$ movable unit point charges at distinct points in $[0, \infty)$. The state of equilibrium in the presence of an additional external potential $v(x)=x$ is now reached at the zeros of the Laguerre polynomial $L_{n}^{(\alpha)}(x)$ provided that the point charges interact according to a logarithmic potential. Stieltjes [24], [25] stated evaluations of the discriminants of the classical orthogonal polynomials of Hermite, Laguerre and Jacobi and his results explicitly give the minimum energy for the Stieltjes electrostatic model, which is the energy of the system at the equilibrium position. Later Hilbert [11] proved Stieltjes statements and Schur gave a very elegant proof in [22]. Recently Forrester and Rogers [9] considered similar problems on the unit circle. In a very recent work, Grünbaum [10] gave an electrostatic interpretation of the zeros of the KoornwinderKrall polynomials. The latter polynomials are orthogonal with respect to a measure with an absolutely continuous component supported on $[-1,1]$ and two discrete masses at the end points \pm 1 .

In this paper we extend the Stieltjes models to general orthogonal polynomials. In the latter part of this section we remind the reader of some 
definitions, some basic facts, and recent related results. In Section 2 we shall describe the new model, state and prove our first main results concerning this model. We also give an explicit formula for the total energy at the equilibrium position of our model in terms of the recursion coefficients of orthonormal polynomials associated with the model. This is formula (2.17) and we hope it will have some applications to statistical mechanics. In another work, in collaboration with Yang Chen, formula (2.17) will be analyzed further and combined with the Coulomb fluid method of F. Dyson to discuss certain models in statistical mechanics, a continuation of [7]. In Section 2 we consider an explicit example worked out to independently verify our results. Section 4 contains a derivation of the limiting behavior of the total energy of the $n$ particle system as $n \rightarrow \infty$ in cases of potentials associated with Freud type weights. In Section 3 we comment on electrostatic interpretation for the Freud weights and Selberg-type integrals. We also mention how the differential equation (1.9) gives information on the Bethe Ansatz for general systems of polynomials orthogonal with respect to an absolutely continuous measure supported on an interval. This also relates to systems of nonlinear equations for the zeros such general orthogonal polynomials and extends earlier work of Ahmed, Bruschi, Calegro, Olshantsky and Perelomov [1], and Mehta [18]. The related and later work of Ahmed and Muldoon [2] also contains a detailed bibiolography on the subject.

Let $\left\{p_{n}(x)\right\}$ be polynomials orthonormal with respect to a weight function $w$ supported on $[a, b]$, finite or infinite and $w(x)>0$ for $x \in(a, b)$. In other words

$$
\int_{a}^{b} p_{m}(x) p_{n}(x) w(x) d x=\delta_{m, n} .
$$

We associate with $w(x)$ an external potential $v(x)$,

$$
w(x)=e^{-v(x)}, \quad x \in(a, b) .
$$

We shall normalize $w$ by $\int_{a}^{b} w(x) d x=1$. The initial values and three term recurrence relation of $\left\{p_{n}(x)\right\}$ take the form

$$
\begin{aligned}
p_{0}(x) & =1, \quad p_{1}(x)=\left(x-b_{0}\right) / a_{1}, \\
x p_{n}(x) & =a_{n+1} p_{n+1}(x)+b_{n} p_{n}(x)+a_{n} p_{n-1}(x), \quad n>0 .
\end{aligned}
$$


Assuming $v$ is twice differentiable and convex function on $[a, b]$ we define $A_{n}(x)$ and $B_{n}(x)$ via

$$
\begin{aligned}
A_{n}(x)= & \frac{a_{n} w\left(b^{-}\right) p_{n}^{2}(b)}{b-x}+\frac{a_{n} w\left(a^{+}\right) p_{n}^{2}(a)}{x-a} \\
& +a_{n} \int_{a}^{b} \frac{v^{\prime}(x)-v^{\prime}(y)}{x-y} p_{n}^{2}(y) w(y) d y, \\
B_{n}(x)= & \frac{a_{n} w\left(a^{+}\right) p_{n}(a) p_{n-1}(a)}{x-a}+\frac{a_{n} w\left(b^{-}\right) p_{n}(b) p_{n-1}(b)}{b-x} \\
& +a_{n} \int_{a}^{b} \frac{v^{\prime}(x)-v^{\prime}(y)}{x-y} p_{n}(y) p_{n-1}(y) w(y) d y .
\end{aligned}
$$

In (1.5) and (1.6) it is assumed that

$$
y^{n} \frac{v^{\prime}(x)-v^{\prime}(y)}{x-y} w(y), \quad n=0,1, \ldots,
$$

are integrable over $(a, b)$ and the boundary terms in (1.5) and (1.6) exist. Under the latter assumptions the orthonormal polynomials $p_{n}$ 's satisfy the differential recurrence relation $[3],[4],[5]$,

$$
p_{n}^{\prime}(x)=A_{n}(x) p_{n-1}(x)-B_{n}(x) p_{n}(x),
$$

and the differential second order equation

$$
p_{n}^{\prime \prime}(x)+R_{n}(x) p_{n}^{\prime}(x)+S_{n}(x) p_{n}(x)=0,
$$

where

$$
\begin{aligned}
R_{n}(x):= & -\left[v^{\prime}(x)+\frac{A_{n}^{\prime}(x)}{A_{n}(x)}\right] \\
S_{n}(x):= & B_{n}^{\prime}(x)-B_{n}(x) \frac{A_{n}^{\prime}(x)}{A_{n}(x)}-B_{n}(x)\left[v^{\prime}(x)+B_{n}(x)\right] \\
& +\frac{a_{n}}{a_{n-1}} A_{n}(x) A_{n-1}(x) .
\end{aligned}
$$

The representations (1.5) and (1.6) of the $A_{n}$ 's and $B_{n}$ 's are in [5] but earlier versions are in $[4]$ and $[3]$.

It is important to note that (1.9) follows from (1.8) and, (1.3) and (1.4), so (1.9) with $R_{n}$ and $S_{n}$ given by (1.10) and (1.11) may hold for polynomials orthogonal with respect to a measure with discrete part. Indeed this is the case, for example, for the Koornwinder polynomials in [14] and [10]. All we need is to eliminate $v^{\prime}$ in (1.10) and (1.11) by using

$$
B_{n}(x)+B_{n+1}(x)=\frac{x-b_{n}}{a_{n}} A_{n}(x)-v^{\prime}(x) .
$$


Observe that $v^{\prime}$ when obtained via (1.12) may depend on $n$ and as such can be thought of as a varying weight. It is clear from (1.3) and (1.4) that

$$
p_{n}(x)=\frac{x^{n}}{a_{1} a_{2} \ldots a_{n}}+\text { lower order terms. }
$$

The discriminant $D_{n}$ of a polynomial $g_{n}$,

$$
g_{n}(x):=\gamma x^{n}+\text { lower order terms, }
$$

is defined by

$$
D_{n}=D\left(g_{n}\right):=\gamma^{2 n-2} \prod_{1 \leq j<k \leq n}\left(x_{j}-x_{k}\right)^{2},
$$

where $x_{1}, x_{2}, \ldots, x_{n}$ are the zeros of $g_{n}$, see [8].

Although we shall not use potential theory in this work, the reader may be interested in consulting Lubinski's publications [15], [16], [17] and the recent book by Saff and Totik [21]. A noteworthy reference is the very influential paper of Nevai [19] on Freud's mathematical legacy.

\section{The Interacting Particle Model.}

We propose that a weight function $w(x)$ creates two external fields. One is a long range field whose potential at a point $x$ is $v(x)$ of (1.2). In addition in the presence of $n$ unit charges $w$ produces a short range field whose potential is $\ln \left(A_{n}(x) / a_{n}\right)$. Thus the total external potential $V(x)$ is the sum of the short and long range potentials, that is

$$
V(x)=v(x)+\ln \left(A_{n}(x) / a_{n}\right) .
$$

The potential energy at $x$ of a point charge $e$ located at $c$ is $-2 e \ln |x-c|$. We shall refer to this potential as a logarithmic potential. Consider the system of $n$ movable unit charges in $[a, b]$ in the presence of the external potential $V(x)$ of $(2.1)$. Let

$$
\mathbf{x}:=\left(x_{1}, x_{2}, \ldots, x_{n}\right),
$$

where $x_{1}, \ldots, x_{n}$ are the positions of the particles arranged in decreasing order. The total energy of the system is

$$
E(\mathbf{x})=\sum_{k=1}^{n} V\left(x_{k}\right)-2 \sum_{1 \leq j<k \leq n} \ln \left|x_{j}-x_{k}\right| .
$$

Let

$$
T(\mathbf{x}):=\exp (-E(\mathbf{x})) .
$$

Theorem 2.1. Assume $w(x)>0, x \in(a, b)$ and let $v(x)$ of (1.2) and $v(x)+\ln A_{n}(x)$ be twice continuously differentiable functions whose second derivative is nonnegative on $(a, b)$. Then the equilibrium position of $n$ movable unit charges in $[a, b]$ in the presence of the external potential $V(x)$ of 
(2.1) is unique and attained at the zeros of $p_{n}(x)$, provided that the particle interaction obeys a logarithmic potential and that $T(\mathbf{x}) \rightarrow 0$ as $\mathbf{x}$ tends to any boundary point of $[a, b]^{n}$, where

$$
T(\mathbf{x})=\left[\prod_{j=1}^{n} \frac{\exp \left(-v\left(x_{j}\right)\right)}{A_{n}\left(x_{j}\right) / a_{n}}\right] \prod_{1 \leq l<k \leq n}\left(x_{l}-x_{k}\right)^{2} .
$$

Before proving Theorem 2.1, observe that finding the equilibrium distribution of the charges in Theorem 2.1 is equivalent to finding the maximum of $T(\mathbf{x})$ in (2.4). The reason is that at interior points of $[a, b]^{n}$, the gradient of $T$ vanishes if and only if the gradient of $E$ vanishes. Furthermore at such points of vanishing gradients the Hessians of $T$ and $E$ have opposite signs. There is no loss of generality in assuming

$$
x_{1}>x_{2}>\cdots>x_{n},
$$

a convention we shall follow throughout this work.

Proof of Theorem 2.1. The assumption $v^{\prime \prime}(x)>0$ ensures the positivity of $A_{n}(x)$. To find an equilibrium position we solve

$$
\frac{\partial}{\partial x_{j}} \ln T(\mathbf{x})=0, \quad j=1,2, \ldots, n .
$$

This system is

$$
-v^{\prime}\left(x_{j}\right)-\frac{A_{n}^{\prime}\left(x_{j}\right)}{A_{n}\left(x_{j}\right)}+2 \sum_{1 \leq k \leq n, k \neq j} \frac{1}{x_{j}-x_{k}}=0, \quad j=1,2, \ldots, n .
$$

Let

$$
f(x):=\prod_{j=1}^{n}\left(x-x_{j}\right)
$$

It is clear that

$$
\begin{aligned}
\sum_{1 \leq k \leq n, k \neq j} \frac{1}{x_{j}-x_{k}} & =\lim _{x \rightarrow x_{j}}\left[\frac{f^{\prime}(x)}{f(x)}-\frac{1}{x-x_{j}}\right] \\
& =\lim _{x \rightarrow x_{j}}\left[\frac{\left(x-x_{j}\right) f^{\prime}(x)-f(x)}{\left(x-x_{j}\right) f(x)}\right]
\end{aligned}
$$

and L'Hôspital's rule implies

$$
2 \sum_{1 \leq k \leq n, k \neq j} \frac{1}{x_{j}-x_{k}}=\frac{f^{\prime \prime}\left(x_{j}\right)}{f^{\prime}\left(x_{j}\right)} .
$$

Now (2.7), (2.8) and (2.9) imply

$$
-v^{\prime}\left(x_{j}\right)-\frac{A_{n}^{\prime}\left(x_{j}\right)}{A_{n}\left(x_{j}\right)}+\frac{f^{\prime \prime}\left(x_{j}\right)}{f^{\prime}\left(x_{j}\right)}=0,
$$


or equivalently

$$
f^{\prime \prime}(x)+R_{n}(x) f^{\prime}(x)=0, \quad x=x_{1}, \ldots, x_{n},
$$

with $R_{n}$ as in (1.10). In other words

$$
f^{\prime \prime}(x)+R_{n}(x) f^{\prime}(x)+S_{n}(x) f(x)=0, \quad x=x_{1}, \ldots, x_{n} .
$$

To check for local maxima and minima consider the Hessian matrix

$$
H=\left(h_{i j}\right), \quad h_{i j}=\frac{\partial^{2} \ln T(\mathbf{x})}{\partial x_{i} \partial x_{j}} .
$$

It readily follows that

$$
\begin{aligned}
h_{i j} & =2\left(x_{i}-x_{j}\right)^{-2}, \quad i \neq j, \\
h_{i i} & =-v^{\prime \prime}\left(x_{i}\right)-\frac{\partial}{\partial x_{i}}\left(\frac{A_{n}^{\prime}\left(x_{i}\right)}{A_{n}\left(x_{i}\right)}\right)-2 \sum_{1 \leq j \leq n, j \neq i} \frac{1}{\left(x_{i}-x_{j}\right)^{2}} .
\end{aligned}
$$

This shows that the matrix $-H$ is positive definite because it is real, symmetric, strictly diagonally dominant and its diagonal terms are positive, $[\mathbf{1 2}$, Cor. 7.2.2]. Therefore $\ln T$ has no relative minima nor saddle points. Thus any solution of (2.10) will provide a local maximum of $\ln T$ or $T$. There cannot be more than one local maximum since $T(\mathbf{x}) \rightarrow 0$ as $\mathbf{x} \rightarrow$ any boundary point along a path in the region defined in (2.6). Thus the system (2.7) has at most one solution. On the other hand (1.9) and (2.11) show that the zeros of

$$
f(x)=a_{1} a_{2} \ldots, a_{n} p_{n}(x),
$$

satisfy $(2.7)$, hence the zeros of $p_{n}(x)$ solve (2.7). This completes the proof of Theorem 2.1.

Let

$$
x_{1 n}>x_{2 n}>\cdots>x_{n n}
$$

be the zeros of $p_{n}(x)$. In [13] we used an idea from Schur [22] to prove that the discriminant of $p_{n}(x)$ is given by

$$
D_{n}=\left\{\prod_{j=1}^{n} \frac{A_{n}\left(x_{j n}\right)}{a_{n}}\right\}\left[\prod_{k=1}^{n} a_{k}^{2 k-2 n+2}\right] .
$$

Our next result gives a representation for the maximum value of $T$ in terms of the recursion coefficients $\left\{a_{n}\right\}$. 
Theorem 2.2. Let $T_{\max }$ and $E_{n}$ be the maximum value of $T(\mathbf{x})$ and the equilibrium energy of the $n$ particle system. Then

$$
\begin{aligned}
T_{\max } & =\exp \left(-\sum_{j=1}^{n} v\left(x_{j n}\right)\right) \prod_{k=1}^{n} a_{k}^{2 k}, \\
E_{n} & =\sum_{j=1}^{n} v\left(x_{j n}\right)-2 \sum_{j=1}^{n} j \ln a_{j} .
\end{aligned}
$$

Proof. Since $T_{\max }$ is

$$
\left[\prod_{j=1}^{n} \frac{\exp \left(-v\left(x_{j n}\right)\right)}{A_{n}\left(x_{j n}\right) / a_{n}}\right] \gamma^{2-2 n} D_{n}\left(p_{n}\right),
$$

then (2.16) follows from (1.15) and (2.15). We also used $\gamma a_{1} \cdots a_{n}=1$. Now (2.17) holds because $E_{n}$ is $-\ln \left(T_{\max }\right)$. This completes the proof.

It is important to observe that if we only know the differential recurrence relation (1.8) and the pure three term recurrence relation (1.3)-(1.4) then using (1.12) Theorem 2.1 can be recast in the following form:

Theorem 2.3. Let $\left\{p_{n}(x)\right\}$ be orthonormal with respect to a positive measure supported on $[a, b]$ and assume that $A_{n}(x)>0$ on $(a, b)$. Define $v^{\prime}$, which may depend on $n$ (up to an additive constant), through (1.12). If $v+\ln \left(A_{n}(x) / a_{n}\right)$ is convex then the equilibrium position described in Theorem 2.1 is unique and is attained at the zeros of $p_{n}(x)$. Furthermore the minimum energy is given by (2.17).

Theorem 2.4. Theorem 2.1 holds if instead of $v^{\prime \prime}(x)>0$ we require $A_{n}(x)$ to have a fixed sign in $(a, b)$ and $A_{n}(x)$ in (2.1) and (2.4) is replaced by $\left|A_{n}(x)\right|$.

Proof. The assumption $v^{\prime \prime}(x)>0$ was only used to guarantee the positivity of $A_{n}(x)$ in $(a, b)$. The rest of the proof remains the same. In the case of Laguerre polynomials $[\mathbf{1 3}]$

$$
w(x)=\frac{x^{\alpha} \exp (-x)}{\Gamma(\alpha+1)}, \quad \frac{A_{n}(x)}{a_{n}}=\frac{1}{x},
$$

and Stieltjes electrostatic interpretation of the zeros of Laguerre polynomials follows from Theorem 2.1. For the Jacobi polynomials

$$
w(x)=\frac{(1-x)^{\alpha}(1+x)^{\beta} \Gamma(\alpha+\beta+2)}{2^{\alpha+\beta+1} \Gamma(\alpha+1) \Gamma(\beta+1)}, \quad \frac{A_{n}(x)}{a_{n}}=\frac{\alpha+\beta+1+2 n}{1-x^{2}},
$$

[13]. This gives Stieltjes result concerning the electrostatics of zeros of Jacobi polynomials. Observe that the assumption $T(\mathbf{x}) \rightarrow 0$ as $\mathbf{x}$ tends to 
a boundary point of $[0, \infty]^{n}$ in the Laguerre case, or a boundary point of $[-1,1]^{n}$ in the Jacobi case is automatically satisfied.

The quantity $E_{n}$ is related to what was denoted by $F_{n}$ in [7]. The first sum in (2.17) is twice the interaction energy while $E_{n}$ is the free energy, [7].

It is worth pointing out that Theorems 2.2 and 2.3 give closed forms for the energy at the equilibrium position in terms of the recursion coefficients. This gives an exactly solvable model in contrast with the earlier models where the location of the moving charges is only found approximately and has no analytic expression other than being the equilibrium position (Fekete points). In a future work we will show that for large $n$ the first two terms in the asymptotics of the energy $E_{n}$ agrees with the corresponding terms of the free energy of the system [7] in several models.

Let us consider the example

$$
w(x)=\frac{2 \exp \left(-x^{4}\right)}{\Gamma(1 / 4)}, \quad \text { or } \quad v(x)=x^{4}+\ln (\Gamma(1 / 4))-\ln 2 .
$$

In the case we have the Freud nonlinear recurrences

$$
n=4 a_{n}^{2}\left(a_{n-1}^{2}+a_{n}^{2}+a_{n+1}^{2}\right),
$$

[19] and $A_{n}(x)$ is given by, [5],

$$
A_{n}(x)=4 a_{n}\left[x^{2}+a_{n}^{2}+a_{n+1}^{2}\right] .
$$

In $(2.22) a_{0}:=0$. We now consider the case $n=3$, so

$$
a_{1} a_{2} a_{3} p_{3}(x)=x\left(x^{2}-a_{1}^{2}-a_{2}^{2}\right) .
$$

Since $v$ is even then the unknowns in (2.7) are $\pm x_{1}$ and zero. The only information we get from $(2.7)$ is that $x_{1}$ must satisfy

$$
4 x^{3}+\frac{2 x}{x^{2}+a_{3}^{2}+a_{4}^{2}}=\frac{3}{x} \text {. }
$$

From (2.22) we get

$$
a_{2}^{2}+a_{3}^{2}+a_{4}^{2}=\frac{3}{4 a_{3}^{2}}=\frac{3 a_{2}^{2}}{2\left[1-2 a_{2}^{2}\left(a_{1}^{2}+a_{2}^{2}\right)\right]} .
$$

Now (2.22) gives $a_{1}^{2}+a_{2}^{2}=1 /\left(4 a_{1}^{2}\right)$ and (2.25) becomes

$$
4 x^{4}+\frac{2 x^{2}}{x^{2}-\frac{1}{4 a_{1}^{2}}+\frac{2 a_{1}^{2}}{12 a_{1}^{4}-1}}=\frac{3}{x},
$$

and it can be factored as

$$
\left(x^{2}-\frac{1}{4 a_{1}^{2}}\right)\left(2 x^{4}+\frac{4 a_{1}^{2} x^{2}}{12 a_{1}^{4}-1}+\frac{1}{12 a_{1}^{4}-1}-\frac{1}{2}\right)=0 .
$$

Clearly (2.24) has solutions $\pm\left(2 a_{1}\right)^{-1}$ which are $\pm \sqrt{a_{1}^{2}+a_{2}^{2}}$, as predicted by Theorem 2.1 and the definition of $p_{3}$ in (2.24). To see that (2.24) has no other 
real solutions it suffices to show $12 a_{1}^{4}-1<2$ which will make the second factor on left-hand side of (2.24) strictly positive. Now $\int_{-\infty}^{\infty} p_{1}^{2}(x) w(x) d x=1$ and $p_{1}(x)=x / a_{1}$ give $a_{1}^{2}=\Gamma(3 / 4) / \Gamma(1 / 4)$ and all we need to show is equivalent to

$$
\frac{4 \Gamma^{2}(7 / 4)}{9 \Gamma^{2}(5 / 4)}<1
$$

But $\Gamma(7 / 4)(=0.91906)$ and $\Gamma(5 / 4)(=0.90640)$ are nearly equal, so $(2.25)$ holds and we have no other real solutions of (2.24) other than $\pm \sqrt{a_{1}^{2}+a_{2}^{2}}$.

\section{Remarks.}

It is known that the zeros $\left\{x_{j n}\right\}$ of the Hermite polynomials satisfy the Bethe Ansatz:

$$
x_{j n}=\sum_{1 \leq k \leq n, k \neq j} \frac{1}{x_{j n}-x_{k n}} .
$$

A similar property also holds for the Jacobi and Laguerre polynomials, [1], $[\mathbf{2}]$. We now show that this property has analogues for general polynomials orthogonal with respect to a weight function. Let $\left\{x_{j n}\right\}$ be the zeros of $p_{n}(x)$. One can express the sums $\sum_{1 \leq k \leq n, k \neq j}\left(x_{j n}-x_{k n}\right)^{-i}$ for fixed $j$ in terms of the values of $v^{\prime}, A_{n}, B_{n}$ and their derivatives at the zeros $\left\{x_{j n}\right\}$. For $i=1$, use (2.9) and (2.10) to get

$$
2 \sum_{1 \leq k \leq n, k \neq j} \frac{1}{x_{j n}-x_{k n}}=v^{\prime}\left(x_{j n}\right)+\frac{A_{n}^{\prime}\left(x_{j n}\right)}{A_{n}\left(x_{j n}\right)} .
$$

For Hermite polynomials (3.2) reduces to (3.1) since $v(x)=x^{2}$ and $A_{n}^{\prime}(x)=$ 0 . As an example consider the Freud weight $w(x)=\exp \left(-x^{4}\right)$ so that $v(x)=x^{4}$. Here $A_{n}(x)$ is $4 a_{n}\left(x^{2}+a_{n}^{2}+a_{n+1}^{2}\right)$ and (3.2) becomes

$$
\sum_{1 \leq k \leq n, k \neq j} \frac{1}{x_{j n}-x_{k n}}=2 x_{j n}^{3}+\frac{x_{j n}}{x_{j n}^{2}+a_{n}^{2}+a_{n+1}^{2}} .
$$

To find $\sum_{1 \leq k \leq n, k \neq j}\left(x_{j n}-x_{k n}\right)^{-2}$ in the general case we use

$$
\begin{aligned}
\sum_{1 \leq k \leq n, k \neq j} \frac{1}{\left(x_{j n}-x_{k n}\right)^{2}} & =\lim _{x \rightarrow x_{j n}}\left[-\frac{d}{d x} \frac{p_{n}^{\prime}(x)}{p_{n}(x)}-\frac{1}{\left(x-x_{j n}\right)^{2}}\right] \\
& =\lim _{x \rightarrow x_{j n}}\left[\left(\frac{p_{n}^{\prime}(x)}{p_{n}(x)}\right)^{2}-\frac{p_{n}^{\prime \prime}(x)}{p_{n}(x)}-\frac{1}{\left(x-x_{j n}\right)^{2}}\right] .
\end{aligned}
$$


After some simplification we get

$$
\sum_{1 \leq k \leq n, k \neq j} \frac{1}{\left(x_{j n}-x_{k n}\right)^{2}}=\left[\frac{p_{n}^{\prime \prime}\left(x_{j n}\right)}{2 p_{n}^{\prime}\left(x_{j n}\right)}\right]^{2}-\frac{p_{n}^{\prime \prime \prime}\left(x_{j n}\right)}{3 p_{n}^{\prime}\left(x_{j n}\right)} .
$$

In terms of the coefficients $R_{n}$ and $S_{n}$ in the differential equation (1.9) the above sum is

$$
\sum_{1 \leq k \leq n, k \neq j} \frac{1}{\left(x_{j n}-x_{k n}\right)^{2}}=\frac{1}{4} R_{n}^{2}\left(x_{j n}\right)-\frac{1}{3} R_{n}\left(x_{j n}\right)+\frac{1}{3}\left[R_{n}^{\prime}\left(x_{j n}\right)+S_{n}^{\prime}\left(x_{j n}\right)\right] .
$$

Similarly we can generate sums of higher powers of differences of zeros.

In the remainder of this section we shall concentrate on the case

$$
v(x)=c_{2 m} x^{2 m}+\text { lower order terms, } \quad m=2,3, \ldots,
$$

and $-a=b=\infty$.

Lemma 3.1. If $v$ is as in (3.6), and is convex then $A_{n}(x)$ is of degree $2 m-2$ and has only complex zeros.

The proof follows from (1.5).

Let $z_{1}, z_{2}, \ldots, z_{2 m-2}$ be the zeros of $A_{n}(x)$.

Theorem 3.2. Under the assumptions of Theorem 2.1 the electrostatic system has $n$ movable unit charges, external field

$$
v(x)+\ln \left(2 m c_{2 m}\right)+V_{1}(x),
$$

where $V_{1}$ is the potential due to $2 m-2$ unit charges at $z_{1}, z_{2}, \ldots, z_{2 m-2}$.

Proof. Clearly (1.5) implies

$$
A_{n}(x) / a_{n}=2 m c_{2 m} \prod_{j=1}^{2 m-2}\left(z-z_{j}\right)
$$

The electrostatic interpretation now follows from (1.5) and (3.8).

Selberg [23] proved

$$
\begin{array}{r}
\int_{[0,1]^{n}}\left\{\prod_{j=1}^{n} t_{j}^{x-1}\left(1-t_{j}\right)^{y-1}\right\} \prod_{1 \leq i<k \leq n}\left|t_{i}-t_{k}\right|^{2 z} d t_{1} \ldots d t_{n} \\
=\prod_{j=1}^{n} \frac{\Gamma(x+(n-j) z) \Gamma(y+(n-j) z) \Gamma(j z+1)}{\Gamma(x+y+(2 n-j-1) z) \Gamma(z+1)}
\end{array}
$$

for $\operatorname{Re}(x)>0, \operatorname{Re}(y)>0$, and $\operatorname{Re}(z)>-\min \{1 / n, \operatorname{Re}(x) / n-1, \operatorname{Re}(y) /(n-$ $1)\}$. Here $[0,1]^{n}$ is the unit cube in $R^{n}$. This integral is the multivariate generalization of the beta integral and is now called the Selberg integral. It is important to note that if we normalize the Jacobi polynomials to be 
orthogonal on $[0,1]$ then the Stieltjes-Hilbert results provide the $L_{\infty}$ norm of

$$
\left\{\prod_{j=1}^{n} t_{j}^{\alpha}\left(1-t_{j}\right)^{\beta}\right\} \prod_{1 \leq i<k \leq n}\left|t_{i}-t_{k}\right|^{2} .
$$

On the other hand the Selberg integral (3.9) essentially gives the $L_{p}$ norm of the expression in (3.10). One is then led to view the Stieltjes-Hilbert results as limiting cases of the Selberg integral. Our Theorem 2.2 extends the Stieltjes-Hilbert results from Jacobi polynomials to general orthogonal polynomials, so it would be of interest to explore the analogue of the Selberg integral and evaluate the integrals

$$
\int_{[a, b]^{n}}\left(\prod_{j=1}^{n} \frac{\exp \left(-v\left(t_{j}\right)\right)}{A_{n}\left(t_{j}\right) / a_{n}}\right)^{p} \prod_{1 \leq i<k \leq n}\left(t_{i}-t_{k}\right)^{2 p} d t_{1} \ldots d t_{n} .
$$

In particular for $v(x)$ as in (2.21) the integral in (3.11) is

$$
\int_{R^{n}} \frac{\exp \left(-p \sum_{j=1}^{n} t_{j}^{4}\right)}{\prod_{j=1}^{n}\left(t_{j}^{2}+a_{n}^{2}+a_{n+1}^{2}\right)^{p}} \prod_{1 \leq i<k \leq n}\left(t_{i}-t_{k}\right)^{2 p} d t_{1} \ldots d t_{n} .
$$

The $a_{n}$ 's can be generated from (2.22) with the initial values

$$
a_{0}=0, \quad a_{1}^{2}=\frac{\Gamma(3 / 4)}{\Gamma(1 / 4)}, a_{2}^{2}=\frac{\Gamma(5 / 4)}{\Gamma(3 / 4)}-\frac{\Gamma(3 / 4)}{\Gamma(1 / 4)} .
$$

It must be emphasized that the $a_{n}$ 's in (3.12) are not arbitrary but are the recursion coefficients of the Freud polynomials.

\section{Energy Asymptotics.}

In this section we first discuss the large $n$ asymptotics of $E_{n}$ in the potential model of the Freud weights. We use the approximation

$$
\sum_{j=1}^{n} v\left(x_{j n}\right) \sim \int_{x_{n n}}^{x_{1 n}} v(x) \sigma(x) d x, \quad n \rightarrow \infty,
$$

where $\sigma(x)$ is the density of the zeros. In (4.1) $f_{n} \sim g_{n}$ as $n \rightarrow \infty$ means $f_{n} / g_{n} \rightarrow 1$ as $n \rightarrow \infty$. In the literature on potential theory the measure $\sigma(x) d x$ is called the equilibrium measure. Consider the Freud weight function

$$
w_{F}(x ; \alpha):=\frac{\exp \left(-|x|^{\alpha}\right)}{2 \Gamma(1+1 / \alpha)}, \quad \alpha \geq 1
$$


This case is well studied in the literature but we will use the special form of $\sigma$ from $[\mathbf{6}]$ because we need the same normalization. We have

$$
\sigma=\sigma(x, \alpha)=\frac{\alpha}{\pi} \frac{2^{1-\alpha} \Gamma(\alpha)}{[\Gamma(\alpha / 2)]^{2}} b^{\alpha-2} \sqrt{b^{2}-x^{2}}{ }_{2} F_{1}\left(1-\alpha / 2,1 ; 3 / 2 ; 1-(x / b)^{2}\right),
$$

where

$$
b:=x_{1 n}=-x_{n n} .
$$

Furthermore from the Freud conjectures

$$
b^{\alpha} \sim \frac{[\Gamma(\alpha / 2)]^{2} 2^{\alpha-1} n}{\Gamma(\alpha)} .
$$

The $A_{n}(x)$ is now defined by (1.5) with vanishing boundary terms. Using the beta function integral [20], the fact

$$
(a)_{n}=\Gamma(a+n) / \Gamma(a),
$$

and the Gauss sum for a hypergeometric function of unit argument, [20], we get

$$
\begin{aligned}
& \int_{-b}^{b} \sigma(x ; \alpha) v(x) d x \\
&=\frac{\alpha}{\pi} \frac{2^{2-\alpha} \Gamma(\alpha)}{[\Gamma(\alpha / 2)]^{2}} b^{2 \alpha} \\
& \quad \cdot \int_{0}|x|^{\alpha} \sqrt{1-x^{2}}{ }_{2} F_{1}\left(1-\alpha / 2,1 ; 3 / 2 ; 1-x^{2}\right) d x \\
&=\frac{\alpha}{\pi} \frac{2^{1-\alpha} \Gamma(\alpha)}{[\Gamma(\alpha / 2)]^{2}} b^{2 \alpha} \sum_{k=0}^{\infty} \frac{(1-\alpha / 2)_{k}}{(3 / 2)_{k}} \int_{0}^{1} t^{(\alpha-1) / 2}(1-t)^{k+1 / 2} d t \\
&=\frac{\alpha}{\pi} \frac{2^{1-\alpha} \Gamma(\alpha)}{[\Gamma(\alpha / 2)]^{2}} b^{2 \alpha} \sum_{k=0}^{\infty} \frac{(1-\alpha / 2)_{k} \Gamma(k+3 / 2) \Gamma((\alpha+1) / 2)}{(3 / 2)_{k} \Gamma(k+2+\alpha / 2)} \\
&=\frac{2^{1-\alpha} \Gamma(3 / 2) \Gamma(\alpha+1) \Gamma((\alpha+1) / 2)}{\pi \Gamma^{2}(\alpha / 2) \Gamma(2+\alpha / 2) b^{-2 \alpha}} F_{1}(1-\alpha / 2,1 ; 2+\alpha / 2 ; 1) \\
&= \frac{2^{1-\alpha} b^{2 \alpha} \Gamma(\alpha) \Gamma((\alpha+1) / 2)}{\sqrt{\pi} \alpha \Gamma^{3}(\alpha / 2)} .
\end{aligned}
$$


The above calculation and the duplication formula for the gamma function and (4.5) give

$$
\sum_{j=1}^{n} v\left(x_{j n}\right) \sim \int_{-b}^{b} \sigma(x ; \alpha) v(x) d x \sim \frac{n^{2}}{\alpha},
$$

as $n \rightarrow \infty$. It is also known that $a_{n} \sim b / 2$. Therefore (4.5) gives

$$
\sum_{j=1}^{n} j \ln a_{j} \sim \frac{1}{2} n^{2} \ln n
$$

and we have proved

$$
E_{n} \sim-\frac{n^{2}}{\alpha} \ln n
$$

This is the same as the main term in the large $n$ form of the free energy in [7]. More precise asymptotics will be developed in a future work. In some cases it is possible to express $E_{n}$ entirely in terms of the recursion coefficients. This is certainly the case when $v(x)=x^{2 m}$ and $m$ is a positive integer. We illustrate this for $v(x)=x^{4}$. Since $v$ is even the $p_{n}$ 's satisfy $p_{n}(-x)=(-)^{n} p_{n}(x)$, so that (1.12) imply

$$
a_{1} a_{2} \ldots a_{n} p_{n}(x)=x^{n}-c_{n, 1} x^{n-2}+c_{n, 2} x^{n-4}+\cdots .
$$

From (1.3) and (1.4) it follows that

$$
c_{n+1,1}=c_{n, 1}+a_{n}^{2} \quad \text { and } \quad c_{n+1,2}=c_{n, 2}+a_{n}^{2} c_{n-1,1},
$$

hence

$$
c_{n, 1}=\sum_{j=1}^{n-1} a_{j}^{2}, \quad \text { and } \quad c_{n, 2}=\sum_{k=3}^{n-1} a_{k}^{2}\left(\sum_{j=1}^{k-2} a_{j}^{2}\right)
$$

Now

$$
\sum_{j=1}^{n} v\left(x_{j n}\right)=\sum_{j=1}^{n} x_{j n}^{4}=\left[\sum_{j=1}^{n} x_{j n}^{2}\right]^{2}-2 \sum_{1 \leq j<k \leq n} x_{j n}^{2} x_{k n}^{2} .
$$

Formula (4.10) and $p_{n}(-x)=(-1)^{n} p_{n}(x)$ imply

$$
c_{n, 1}=\sum_{j=1}^{n} x_{j n}^{2}, \quad c_{n, 2}=\sum_{1 \leq j<k \leq n} x_{j n}^{2} x_{k n}^{2},
$$

hence

$$
E_{n}=c_{n, 1}^{2}-2 c_{n, 2}-2 \sum_{k=1}^{n} k \ln a_{k},
$$

and $c_{n, 1}$ and $c_{n, 2}$ are given by (4.12). 


\section{Acknowledgments.}

Thanks to Richard Askey whose enthusiastic lectures on the Stieltjes-Hilbert electrostatic models and Selberg integrals introduced me to the subject and for his comments on this manuscript. Thanks also to Walter Van Assche who kindly verified that the equilibrium position of Freud weight with $\alpha=4$ is indeed attained at the zeros of the Freud polynomials for several $n$ 's using his wonderful computer program. I am indebted to a very careful referee who repeated the calculations and pointed out several slips. I grateful to Joseph Keller for his interest and criticisms which improved this manuscript.

\section{References}

[1] S. Ahmed, M. Bruschi, F. Calegro, M.A. Olshantsky and A.M. Perelomov, Properties of the zeros of the classical orthogonal polynomials and of the Bessel functions, Nuovo Cimento, 49B (1979), 173-199.

[2] S. Ahmed and M. Muldoon, Reciprocal power sums of differences of zeros of special functions, SIAM J. Math. Anal., 14 (1983), 372-382.

[3] W. Bauldry, Estimates of asymmetric Freud polynomials on the real line, J. Approximation Theory, 63 (1990), 225-237.

[4] S.S. Bonan and D.S. Clark, Estimates of the Hermite and the Freud polynomials, J. Approximation Theory, 63 (1990), 210-224.

[5] Y. Chen and M.E.H. Ismail, Ladder operators and differential equations for orthogonal polynomials, J. Phys., A30 (1997), 7818-7829.

[6] - Hermitean matrix ensembles and orthogonal polynomials, Studies in Appl. Math., 100 (1998), 33-52.

[7] Y. Chen, M.E.H. Ismail and W. Van Assche, Tau-function constructions of the recurrence coefficients of orthogonal polynomials, Advances in Appl. Math., 20 (1998), 141-168.

[8] L.E. Dickson, New Course on the Theory of Equations, Wiley, New York, 1939.

[9] P.J. Forrester and J.B. Rogers, Electrostatics and the zeros of the classical orthogonal polynomials, SIAM J. Math. Anal., 17 (1986), 461-468.

[10] F.A. Grünbaum, Variation on a theme of Stieltjes and Heine, J. Comp. Appl. Math., 99 (1998), 189-194.

[11] D. Hilbert, Über die Discriminante der in Endlichen abbrechenden hypergeometrischen Reihe, J. für die reine und angewandte Matematik, 103 (1885), 337-345.

[12] R.A. Horn and C. Johnson, Matrix Analysis, Cambridge University Press, Cambridge, 1992.

[13] M.E.H. Ismail, Discriminants and functions of the second kind of orthogonal polynomials, Results in Mathematics, 34 (1998), 132-149.

[14] T.H. Koornwinder, Orthogonal polynomials with weight function $(1-x)^{\alpha}(1+x)^{\beta}+$ $M \delta(x+1)+N \delta(x-1)$, Canadian Math. Bull., 27 (1984), 205-214.

[15] D.S. Lubinsky, A survey of general orthogonal polynomials for weights on finite and infinite intervals, Acta Applicandae Mathematicae, 10 (1987), 237-296. 
[16] , Strong Asymptotics for Extremal Errors and Polynomials Associated with Erdös-Type Weights, Pitman Research Notes in Mathematics, Vol. 202, Longman, Harlow, 1989.

[17] _ An update on orthogonal polynomials and weighted approximation on the real line, Acta Applicandae Mathematicae, 33 (1993), 121-164.

[18] M.L. Mehta, Properties of the zeros of a polynomial satisfying a second order linear partial differential equation, Lett. Nuovo Cimento, 26 (1979), 361-362.

[19] P. Nevai, Géza Freud, orthogonal polynomials and Christoffel functions: A case study, J. Approx. Theory, 48 (1986), 3-167.

[20] E.D. Rainville, Special Functions, Chelsea, Bronx, 1971.

[21] E.B. Saff and V. Totik, Logarithmic Potentials With External Fields, Springer-Verlag, New York, 1997.

[22] I. Schur, Affektlose Gleichungen in der Theorie der Laguerreschen und Hermiteschen Polynomes, J. für die reine und angewandte Matematik, 165 (1931), 52-58.

[23] A. Selberg, Bemerkninger om et multiplet integral, Norsk Mat. Tidsskr., 26 (1944), 71-78.

[24] T.J. Stieltjes, Sur quelques théorèmes d'algèbre, Comptes Rendus de l'Academie des Sciences, Paris, 100 (1885), 439-440; Oeuvres Complètes, Vol. 1, 440-441.

[25] _ Sur les polynômes de Jacobi, Comptes Rendus de l'Academie des Sciences, Paris, 100 (1885), 620-622; Oeuvres Complètes, Vol. 1, 442-444.

[26] G. Szegö, Orthogonal Polynomials, Fourth Edition, Amer. Math. Soc., Providence, 1975.

Received March 24, 1998. This research was partially supported by NSF grant DMS9625459 .

University of South FloridA

TAMPA, FL 33620-5700

E-mail address: ismail@math.usf.edu 